\title{
A unilateral Dentigerous cyst associated with impacted mandibular canine crossing the midline: a rare case report.
}

\author{
${ }^{1}$ Fareed Ahmed Bava, ${ }^{2}$ Abdul kalam Azad, ${ }^{3}$ Mohammed farhan, \\ ${ }^{4}$ Salman siddeeqh
}

\begin{abstract}
:
Introduction: A dentigerous cyst is the most common developmental odontogenic cyst and is frequently noted as an incidental finding on radiographs. The most common teeth affected are impacted mandibular third molars and permanent maxillary canines. This case involves a dentigerous cyst encompassing the right impacted mandibular canine and crossing the midline. This is a rare case of dentigerous cyst encompassing non-adjacent teeth and crossing the midline reported in recent literature.

Case presentation: The patient presented to our department of oral and maxillofacial surgery for treatment of swelling in the lower chin area. The patient was a 10-year old girl with a dentigerous cyst extending from right to left mandibular canines and crossing the midline with the right mandibular canine being impacted.

Conclusion: This case involves an unusual clinical and radiographic presentation of a dentigerous cyst crossing midline. It shows a new variant of presentation that dentists, especially maxillofacial surgeons, paedodontists and radiologists, should be aware of, since a dentigerous cyst crossing the midline is rare.
\end{abstract}

\section{Introduction:}

Dentigerous cysts are the second most common developmental odontogenic cysts. They are frequently noted as an incidental finding on radiographs because a majority of these cysts are asymptomatic and are mostcommonly associated with impacted mandibular third molars and permanent maxillary canines ${ }^{1}$. A dentigerous cyst presents as a well-defined radiolucent entity surrounding the crown of an impacted tooth. The border of the cyst is continuous with the cemento-enamel junction of the impacted tooth. This radiographic finding is pathognomonic for a dentigerous cyst. Majority of the reported cases to date have involved cysts localized to one quadrant of the jaws and have encompassed adjacent teeth. As a dentigerous cyst enlarges, it displaces the affected tooth or teeth apically. Dentigerous cysts enlarge as a result of the accumulation of fluid between the crown of an unerupted tooth and the reduced enamel epithelium. ${ }^{2}$ Migration of an impacted tooth across the midline is a rare anomaly. Transmigration is defined as 'migration of impacted tooth from its normal position to contralateral hemi arch. Ando et al were the first to use the term 'transmigration'. The transmigrated teeth may be impacted in a horizontal or semi-horizontal position. The dentigerous cyst encloses the crown of an un-erupted tooth and is attached to the tooth at the cemento-enamel junction. This cyst develops by accumulation of fluid between the reduced enamel epithelium and the tooth crown. Here we present a case of dentigerous cyst associated with transmigrated impacted right mandibular canine. ${ }^{3}$

\section{Case Presentation}

A patient of 10-year-old girl presented to the dental clinic for swelling of lower chin. A digital pantomograph and occlussal radiographs were made. The pantomograph showed impacted right mandibular canine which was inclined distally in a nearly horizontal position and located in the region apical to the mandibular incisors. There was a well-defined radiolucent area evident around the crown of the right mandibular canine.The radiolucent area measured approximately $20 \mathrm{~mm}$ in width and $11 \mathrm{~mm}$ in height. The appearance was consistentwith a dentigerous cyst encompassing the crown of right mandibular canine.

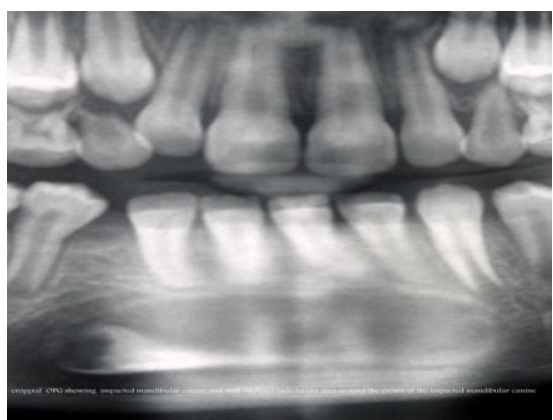

Cropped OPG showing the impacted mandibular canine and a well-defined, radiolucent area around the crown of the impacted mandibular right canine. 
The cyst encompassed the crown and root of the right mandibular canine, extending upto apical portion of left mandibular canine. The border of the dentigerous cyst was continuous with the cemento-enamel junction of the impacted permanent mandibular canines. The appearance was suggestive of a single dentigerous cyst that crossed the midline and encompassed entire impacted right mandibular canine.

Surgical exposure of the canine with an excisional biopsy of the surrounding dentigerous cyst was performed.The excisional biopsy specimen was sent to the department of Oral Pathology for pathologic examination. Hematoxylin and eosin-stained sections of the specimen were prepared.
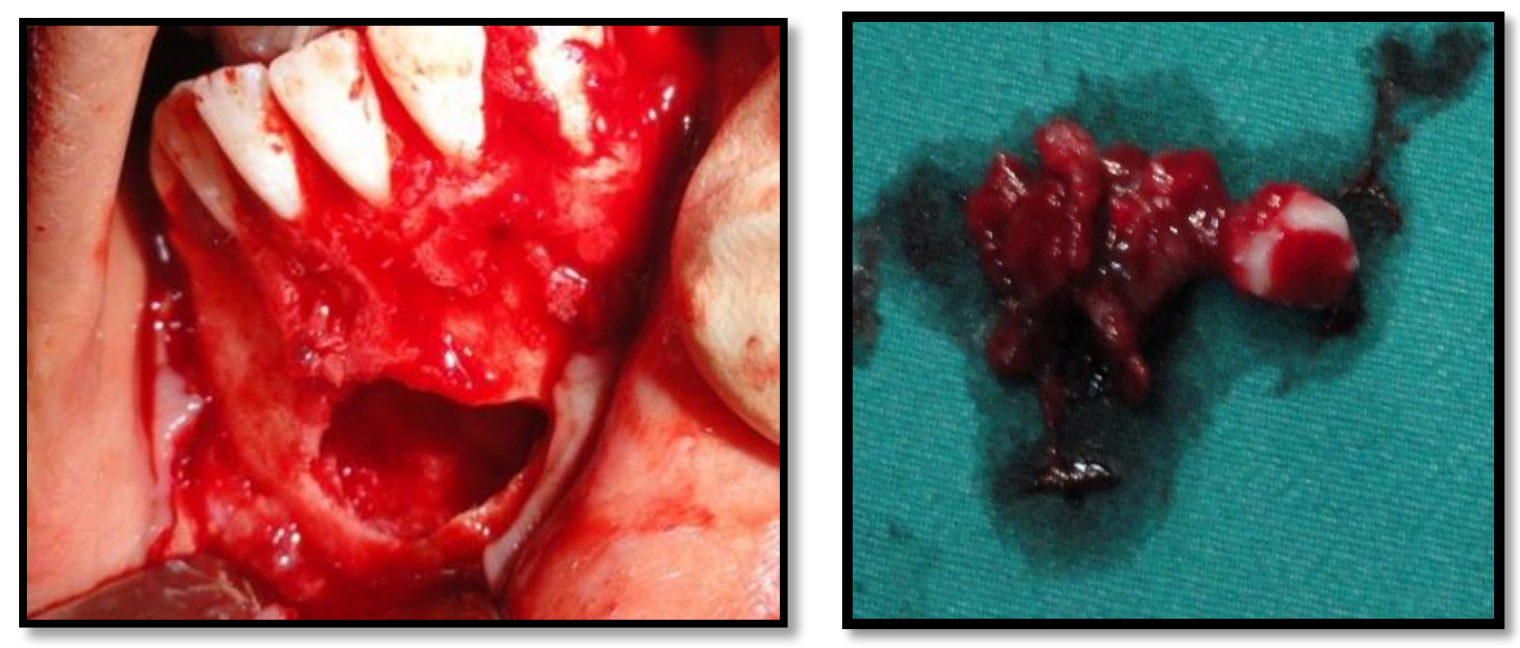

The histopathology report stated that the epithelial lining was non keratinized stratified squamous in type and showed an attachment with the cemento enamel junction. The cystic lining consisted of varying thickness ranging from double to multiple cell layers. The connective tissue wall was fibrous and loosely arranged. Infiltration of Chronic inflammatory cells were seen, with the above findings and correlating with the clinical history a report of dentigerous cyst was given.

\section{Discussion}

The British Standards Institution defines a cyst as 'an abnormal cavity within a tissue, the contents of which may be fluid or semi-fluid, but not pus, at least at the onset', and conventionally the benign cystic lesions occurring in the jaws are subdivided into odontogenic, fissural and bone cysts. Cysts of the jaw present as swellings of jaws and midface. Of the different varieties, odontogenic cysts are the ones that develop from epithelial remnants of the tooth forming-organ. Dentigerous cyst is that type of odontogenic cyst that arises from the reduced enamelorgan as a result of fluid accummulation between its layers. This occurs due to obstruction of the venous outflow as a result of compression of tooth-follicle by the erupting tooth (Main, 1970). Dentigerous cyst is commoner in the $3 \mathrm{rd} \& 4^{\text {th }}$ decades (Shear, 1992). ${ }^{4}$ Our patient was only 10years old at the time of presentation. Dentigerous cyst most commonly develops around the crown of the $3^{\text {rd }}$ mandibular molar tooth. But in our case, right mandibular canine was found to be the cause of the dentigerous cyst and also crossing the midline which is rare of its entity.

In the present case impacted mandibular canines were classified based on angulations and depths of the involved teeth. In terms of angulation impacted mandibular canines can be classified as mesioangular, distoangular, vertical, or horizontal. Depth of the impactions were classified as Level A, Level B, and Level C as follows

Level A.The crown of the impacted caninetooth is at the cervical line of the adjacent teeth.

Level B.The crown of the impacted caninetooth is between the cervical line and rootapices of the adjacent teeth.

Level C.The crown of the impacted canines is beneath the root apices of the adjacent teeth. ${ }^{5}$

Dentigerous cysts are usually solitary, slow growing, asymptomatic lesions that are incidentally found during routine radiographs taken to identify a missing tooth. They can occur at any location of the jaw but frequently seen in relation to impacted mandibular third molars followed by the maxillary canines and maxillary third molars. Occasionally these cysts become painful when infected causing swelling and erythema. The cyst is usually small but when large, results in the expansion and thinning of the cortex leading to pathological fracture. Although the clinical presentations are classical of a dentigerous cyst, in our case it is associated with mandibular canine crossing the midline or transmigrating which is rare in the literature. Radiographic features 
are specific to the lesion characterized by a well defined radiolucency circumscribed by a sclerotic border, associated with the crown of an impacted or unerupted tooth. The borders may be ill-defined when infected. Rarely may they be found with odontoma or a supernumerary tooth. Although they mimic a normal tooth follicle, literatures suggest any follicular space of more than $4 \mathrm{~mm}$ to be a dentigerous cyst. Radiographically the cyst is classified according to its relation with the involved tooth crown as central, lateral and circumferential type. The central type is the most common and presents surrounding the crown. The lateral dentigerous cyst is that, which partially surrounds the crown and extends along the side of the root. The circumferential variant surrounds both the crown and the root of the involved tooth.

The most common treatment modalities for dentigerous cyst are by Enucleation, Marsupialization and decompression of cyst by fenestration. Motamedi et al suggested the criteria for selecting the treatment modality based on the age, size, location, stage of root development, position of the involved tooth and relation of the lesion to the adjacent tooth and vital structure. The most preferred treatment is enucleation with the removal of unerupted or impacted tooth. If the cyst is associated with the canine or premolar with favorable eruptive position, then extraction of the associated tooth is deferred.

Large dentigerous cyst may be treated with marsupialization followed by enucleation. The prognosis is excellent when the cyst is enucleated in toto and recurrence is rare.

\section{Conclusion}

This case describes the presentation of the dentigerous cyst associated with impacted right lower canine crossing the midline which is a rare case in the literature. As the clinical finding, unerupted tooth may present the symptoms of dentigerous cyst, a thorough radiographic evaluation is mandatory for unerupted tooth that have passed their chronological date of eruption and should be promptly attended, to prevent the potential morbidity.

\section{References}

[1]. Daley TD, Wysocki SP, Pringle GA; Relative incidence of odontogenic tumors and oral and Jaw cyst in a Canadian population.OralSug OralMed Oral Pathol 1994;77:276-280

[2]. White Sc, PharoahMJ : Cyst and cyst like lesion of the jaws. In oral Radiology:Principles and Interpretation, 6 edition. Edited by:White SC, Pharoah MJ, St. Louis Mo: Mosby Elsevier:2009;346-350

[3]. International journal of Dental case Report2012;2(1):39-42

[4]. Journal of Indian dental association vol.5, No. 4, April 2011.

[5]. IndianJournal of Multidiciplinary Dentistry, Volume 1, Issue 5, July -August 2011 